\title{
Immunohistochemical features of CD133 expression: Association with resistance to chemoradiotherapy in rectal cancer
}

\author{
SUSUMU SAIGUSA ${ }^{1}$, KOJI TANAKA $^{1}$, YUJI TOIYAMA ${ }^{1}$, TAKESHI YOKOE $^{1}$, YOSHINAGA OKUGAWA ${ }^{1}$, \\ AYA KAWAMOTO ${ }^{1}$, HIROMI YASUDA ${ }^{1}$, YUKI MORIMOTO ${ }^{1}$, HIROYUKI FUJIKAWA ${ }^{1}$, \\ YASUHIRO INOUE $^{1}$, CHIKAO MIKI $^{1}$ and MASATO KUSUNOKI ${ }^{1,2}$
}

Departments of ${ }^{1}$ Gastrointestinal and Pediatric Surgery, and ${ }^{2}$ Innovative Surgery, Division of Reparative Medicine, Institute of Life Sciences, Mie University Graduate School of Medicine, 2-174 Edobashi, Tsu, Mie 514-8507, Japan

Received March 16, 2010; Accepted April 30, 2010

DOI: $10.3892 /$ or_00000865

\begin{abstract}
CD133 has been described as a marker for cancer stem cells (CSCs) in colorectal cancer. Additionally, it has been reported that CSCs are resistant to chemoradiotherapy (CRT). After previously observing that CD133 mRNA levels were elevated after CRT in rectal cancer patients, we further investigated CD133 expression in colorectal cancer following CRT using immunohistochemistry. Forty patients with primary colorectal cancers and 50 patients with rectal cancer who had received preoperative CRT followed by surgery were selected. Sections of formalin-fixed, paraffin-embedded specimens were stained for CD133, CK20 and Ki-67. To clarify the change of CD133 protein after irradiation, CD133 protein levels were examined in radiation-treated human colon cancer cell line HT29. We found four distinct patterns of CD133 staining defined by CD133 expression in luminal surface, in intraluminal cells and in cytoplasm. In total, CD133 expression was detected in $27.5 \%$ of non-CRT and $70 \%$ of CRT specimens. The frequency of CD133 staining in CRT specimens was significantly higher than that of nonCRT specimens. Seven out of 50 CRT specimens exhibited cytoplasmic staining. These cells with cytoplasmic CD133 expression did not express CK20 or Ki-67. The ratio of histopathological responder in cases with CD133 expression in both luminal surface and cytoplasm was significantly lower than that without it $(\mathrm{P}<0.05)$. In vitro study showed that CD133 protein was increased in a radiation-dose dependent manner. Further studies clarifying the role of CD133 in tumor re-growth and resistance to conventional CRT in colo-
\end{abstract}

Correspondence to: Dr Susumu Saigusa, Department of Gastrointestinal and Pediatric Surgery, Division of Reparative Medicine, Institute of Life Sciences, Mie University Graduate School of Medicine, 2-174 Edobashi, Tsu, Mie 514-8507, Japan

E-mail: saigusa@clin.medic.mie-u.ac.jp

Key words: CD133, chemoradiotherapy, colorectal cancer, immunohistochemistry rectal cancer may assist the development of future cancer therapeutics.

\section{Introduction}

Cancer stem cells (CSCs) are a small sub-population of cancer cells that have stem cell-like properties such as selfrenewal and the ability to differentiate into multiple cell types $(1,2)$. CSCs are referred to as tumor-initiating cells (TICs) due to these properties. These TICs have been identified in colorectal cancer as well as in other human malignancies (3).

CD133, also known as prominin-1, is a cell-surface transmembrane glycoprotein which has been used in the identification of putative CSCs from several solid tumors $(4,5)$. O'Brien et al reported that CD133 positive colon cancer TICs were able to maintain themselves as well as differentiate and re-establish tumor heterogeneity upon serial transplantation (1). Ricci-Vitiani et al demonstrated that CD133 positive cells injected into immunodeficient mice formed tumors, whereas CD133 negative cells failed to form tumors (6). This result suggested that CD133-positive cells are much more capable of initiating tumors. Ieta et al investigated CD133 expression in colon cancer cell lines and reported that CD133 positive cells are useful markers for the detection of TICs (7). Thus, in colon cancer, it is widely thought that CD133 is a useful marker for the detection of TICs. Controversially, Shmelkov et al reported that CD133 gene expression was not restricted to cancer stem cells because CD133 was ubiquitously expressed on differentiated colonic epithelium in both adult mice and humans, and that CD133 was widely expressed on human primary colon cancer epithelial cells. Additionally, in human metastatic colon cancer, CD133 expression did not identify most epithelial and TICs (8). Although the function of CD133 has been gradually becoming clear, the significance of CD133 as a CSC marker has been actively studied in recent years.

Recent research suggests that CSCs are particularly resistant to conventional chemoradiotherapy compared with the non-CSC compartment $(9,10)$. For this reason, it was suggested that CD133 positive cells were resistant to CRT by mechanisms including preferential activation of the DNA damage checkpoint response, increased DNA repair capacity, 
production of IL-4 or high expression of anti-apoptosis genes preventing apoptosis (9-11).

In a previous study, we found elevated levels of CD133 mRNA after CRT compared with rectal cancer before CRT $(12,13)$. However, the protein expression pattern of CD133 has not been thoroughly examined in residual cancer cells after CRT. CD133 protein is known to be present at different locations, both in lumina and cytoplasm and it has been reported that cytoplasmic expression of CD133 was found in a minor population of cells (14).

In this study, we attempted to clarify the immunohistological features and the change of CD133 expression in rectal cancer in relation to $\mathrm{CRT}$.

\section{Materials and methods}

Patients and specimens. From 2001 to 2008, 50 patients with rectal cancer who had received preoperative CRT followed by surgery in our institute (criteria for preoperative CRT in our institution; age $<80$ years old, clinical stage II/III based on the International Union Against Cancer's TNM classification, no evidence of distant metastases, no invasion of external sphincter muscle and elevator muscle of anus, and no evidence of deep venous thrombosis) were selected retrospectively. To compare with their patterns of CD133 expression, 40 patients with primary colorectal cancer (clinical stage II/III) were also selected. The CRT regimen included four cycles of 5-fluorouracil (5FU) given as $600 \mathrm{mg} / \mathrm{m}^{2}$ for $24 \mathrm{~h}$ on a continuous intravenous route, and tegafur-uracil (UFT) given as $400 \mathrm{mg} / \mathrm{m}^{2}$ for 5 days orally, with concurrent 20-45 Gy pelvic radiotherapy using a four-field box technique, followed by resection. This regimen was based on the combination of continuous infusion of 5FU and UFT (15). The time interval between preoperative CRT and surgery was two to three weeks. Formalin-fixed paraffinembedded (FFPE) specimens were available for this study. All patients signed informed consent forms for their tissue to be used in this study.

The degree of histopathological tumor regression based on Guidelines for the Clinical and Pathological Studies on Carcinoma of the Colorectum (16) was classified into four categories: grade 0 , neither necrosis nor regressive changes; grade $1 \mathrm{a},>2 / 3$ vital residual tumor cells (VRTCs); grade $1 \mathrm{~b}$, approximately $1 / 3$ to $2 / 3$ VRTCs; grade $2,<1 / 3$ VRTCs; and grade 3 , no VRTCs. We defined grade 2 and 3 as histopathological responders, and defined others as non-responders.

Immunohistochemistry for CD133, CK20 and Ki-67. Two $\mu$ m-thick sections of FFPE specimens were made. We stained more than two different sections per case to confirm reproducibility. After deparaffinization and dehydration, antigen unmasking was accomplished by bringing the specimens to boil in $10 \mathrm{mM}$ sodium citrate buffer. Specimens were then blocked and incubated with primary antibody overnight at $4^{\circ} \mathrm{C}$. Bound antibody was detected by Envision reagents (Envision kit/HRP, Dako Cytomation, Denmark). All sections were counterstained with hematoxylin. Primary anti-CD133 rabbit monoclonal antibody (clone C24B9, Cell Signaling Technology, Denvers, MA, USA), monoclonal mouse anti-human cytokeratin 20 (clone ks20.8, Dako
Cytomation) and monoclonal mouse anti-human Ki-67 antigen (mouse IgG1; Zymed Laboratories, San Francisco, CA, USA) were used at a dilution of 1:100, and detection was by an HRP-labeled streptavidin/biotin method (LASB2 kit/HRP, Dako Cytomation). Negative controls were run simultaneously with preimmune immunoglobulin. Negative controls remained negative for each colorectal cancer.

Cell culture. The human colon cancer cell line HT29 was grown in monolayer cultures in RPMI-1640 (Sigma-Aldrich, Inc., St. Louis, MO, USA) supplemented with fetal bovine serum (FBS, 10\% (v/v), Gibco-BRL, Tokyo, Japan), glutamine $(2 \mathrm{mM})$, penicillin $(100000$ units/l), streptomycin $(100 \mathrm{mg} / \mathrm{l})$, and gentamycin $(40 \mathrm{mg} / \mathrm{l})$ at $37^{\circ} \mathrm{C}$ in a $5 \% \mathrm{CO}_{2}$ environment. For routine passage, cultures were spilt 1:10 when they reached $90 \%$ confluence, generally every 3 days. Cells at the fifth to ninth passage were used for all experiments, which were performed with exponential growing cells.

Radiation exposure. HT-29 cells were plated at a density of $10^{4}$ cells per well in three $100-\mathrm{mm}$ culture dishes (BD Falcon). Exponentially growing cells were plated on a $10-\mathrm{cm}$ dish and irradiated at a dose of 1, 2.5, and 5 Gy (CAX-150-20, Chubu Medical Co., Ltd.). Non-irradiated controls were handled identically to the irradiated cells with the exception of the radiation exposure. After irradiation, cultures were kept at $37^{\circ} \mathrm{C}$ and $5 \% \mathrm{CO}_{2}$ in an incubator.

Clonogenic survival assay after irradiation. Colony formation assays were performed in triplicates. After 10-14 day incubation, cells were fixed for $15 \mathrm{~min}$ with $2 \%$ formaldehyde and stained for $15 \mathrm{~min}$ with $0.5 \%$ crystal violet. Colonies were counted when they contained $>50$ cells. The number of colonies was counted, and then the surviving fraction was normalized to the surviving fraction of the corresponding control.

Western blot analysis. Plates were washed in ice-cold PBS, and cold lysis buffer (Tris-buffered saline, $\mathrm{pH} 7.5$, containing $1 \%$ Triton X-100) was added directly to the plates. Cells were scraped from plates, collected, and homogenized by using a Mixer Mill MM 300 homogenizer (Qiagen Inc., Chatsworth, CA, USA). Supernatants were collected and frozen at $-20^{\circ} \mathrm{C}$ until use. The protein concentration was measured by the BCA protein assay (Pierce, Rockford, IL, USA). Lysate proteins $(20 \mu \mathrm{g})$ were mixed with an equal volume of $2 \mathrm{X}$ Laemmli's loading buffer containing $2 \mathrm{ME}$ and heated at $100^{\circ} \mathrm{C}$ for $5 \mathrm{~min}$. Samples were electrophoretically separated on $12.5 \%$ gradient polyacrylamide gels containing $0.1 \%$ SDS, followed by semi-dry transfer to an Immun-Blot PVDF membrane (Bio-Rad Laboratories, Hercules, CA, USA). The membranes were blocked using 5\% skimmed milk in Tris-buffered saline, $\mathrm{pH} 7.5$, supplemented with $0.1 \%$ Tween-20 (TBS-T). The blots were then incubated with rabbit monoclonal anti-CD133 antibody (Cell Signaling Technology, Inc., Boston, MA) at a 1:1000 dilution and mouse monoclonal anti-actin (clone C4) antibody (MP Biomedicals, LLC, Solon, OH, USA) at a 1:400 dilution in 5\% skimmed milk in TBS-T overnight at $4^{\circ} \mathrm{C}$. After washing three times in TBS-T, the blots were incubated with alkaline- 


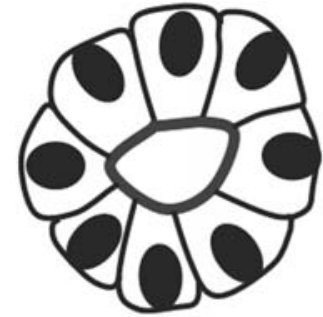

Pattern 1: Luminal surface with/without intraluninal contents

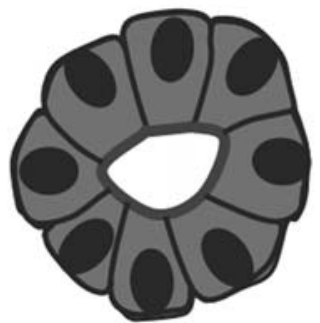

Pattern 3: Luminal surface and cytoplasm

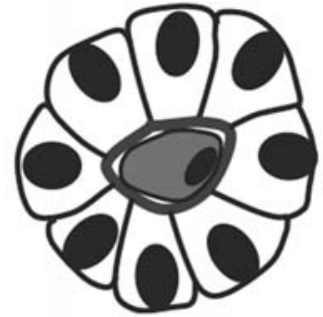

Pattern 2: Luminal surface and intraluminal cell

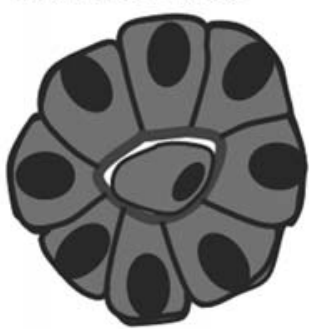

Pattern 4: Luminal surface, cytoplasm, and intraluminal cell
Figure 1. Variation of CD133 staining.

phosphatase-conjugated goat anti-rabbit or mouse $\mathrm{IgG}$ (Promega, Madison, WI, USA) at a 1:200 dilution in 5\% skimmed milk in TBS-T. Following treatment with an enhanced chemiluminescence detection solution, chemiluminescent signals were visualized in a CS Analyzer and AE-6962 light capture (ATTO Corp., Tokyo, Japan).
Statistical analysis. Statistical analysis was done using Stat View 5.0 (SAS Institute Inc., Cary, NC). Significance of difference was analyzed using the $\chi^{2}$ test. P-value $<0.05$ was considered statistically significant.

\section{Results}

Variation of CD133 expression in colon cancer. We found four patterns of CD133 expression in colon cancer specimens as follows: pattern 1, luminal surface with/without intraluminal contents; pattern 2, luminal surface and intraluminal cells; pattern 3, luminal surface including cytoplasm; pattern 4, luminal surface including cytoplasm, and intraluminal cells (Fig. 1).

Table I shows the frequency of CD133 expression according to the luminal surface and cytoplasmic staining patterns. In total, $27.5 \%$ of non-CRT and $70 \%$ of CRT specimens expressed CD133. We defined positive cytoplasmic staining as residual cancer cells that had granules staining positively for CD133 in their cytoplasm. There were no specimens with only cytoplasmic staining. No cytoplasmic staining was found in non-CRT specimens. On the other hand, seven out of 50 specimens after CRT had cytoplasmic staining. Specimens with CRT had higher frequency of CD133 expression (luminal surface; $\mathrm{P}<0.0001$, cytoplasm; $\mathrm{P}=0.0137)$.

Immunohistochemical CD133 expression in residual cancer with CRT. We also observed staining pattern 1 in CRT specimens (Fig. 2A and B). Additionally, we detected staining patterns 2,3 , and 4 present in CRT specimens. Intraluminal

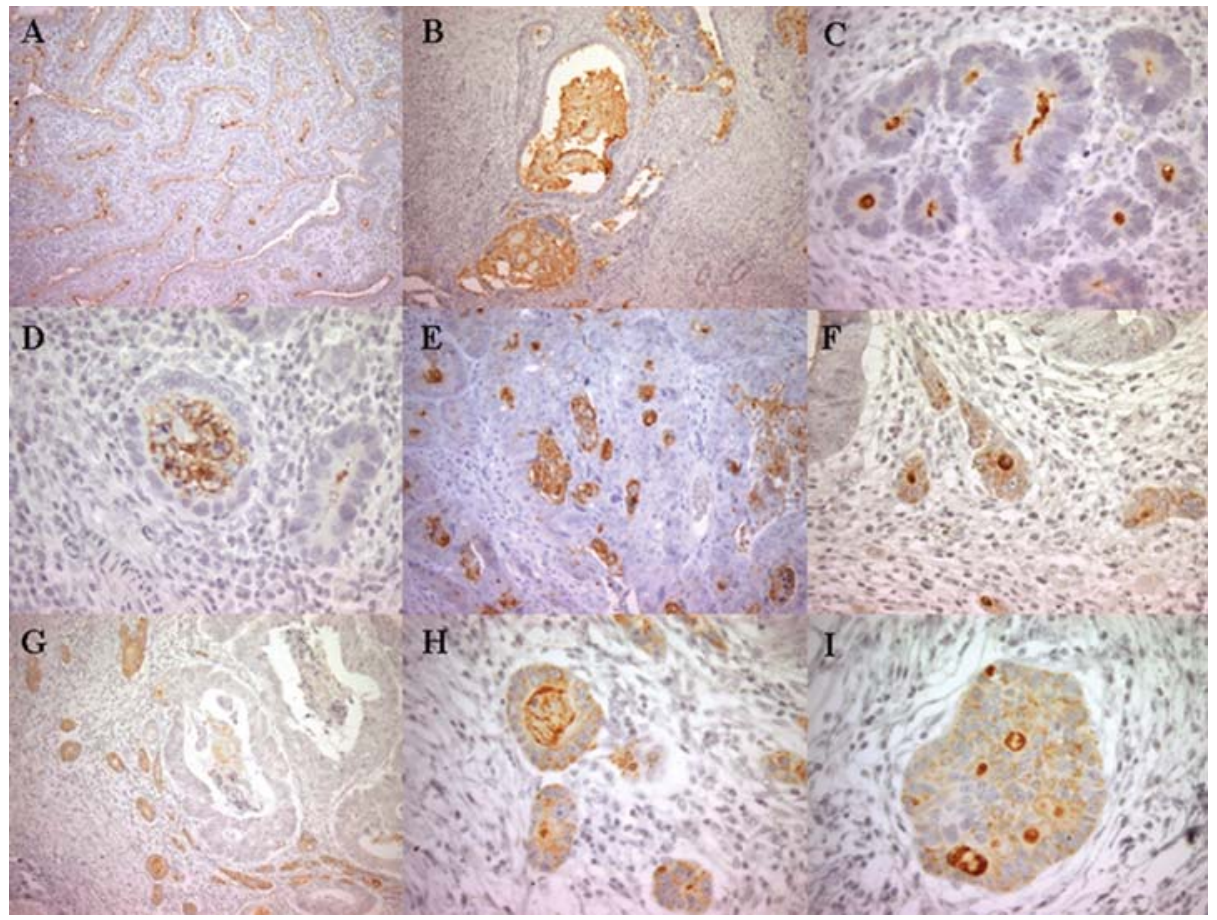

Figure 2. Expression of CD133 in residual cancer specimens with CRT. (A) A similar staining pattern, pattern 1, as seen in the non-CRT specimens, is also seen in CRT specimens. (B and C) CD133 staining in the luminal surface with intraluminal contents is shown. (D and E) Pattern 2 is shown. Intraluminal cells with CD133 staining were detected. (F-I) Patterns 3 and 4 are shown. Cytoplasmic CD133 staining is clearly observed, (I) seems to be a colonosphere. Original magnification, $\mathrm{x} 40$ (A and B), x100 (E and G), x200 (C, D and F), and X400 (H and I). 
Table I. Frequency of CD133 expression in luminal surface and cytoplasm.

\begin{tabular}{lcrr}
\hline & $\begin{array}{c}\text { Non-CRT } \\
(\mathrm{n}=40)\end{array}$ & $\begin{array}{c}\text { CRT } \\
(\mathrm{n}=50)\end{array}$ & P-value \\
\hline Luminal suface (\%) & $11(27.5)$ & $35(70.0)$ & $<0.0001$ \\
Cytoplasm (\%) & $0(0)$ & $7(14.0)$ & 0.0137 \\
\hline$\chi^{2}$ test. & & & \\
\hline
\end{tabular}

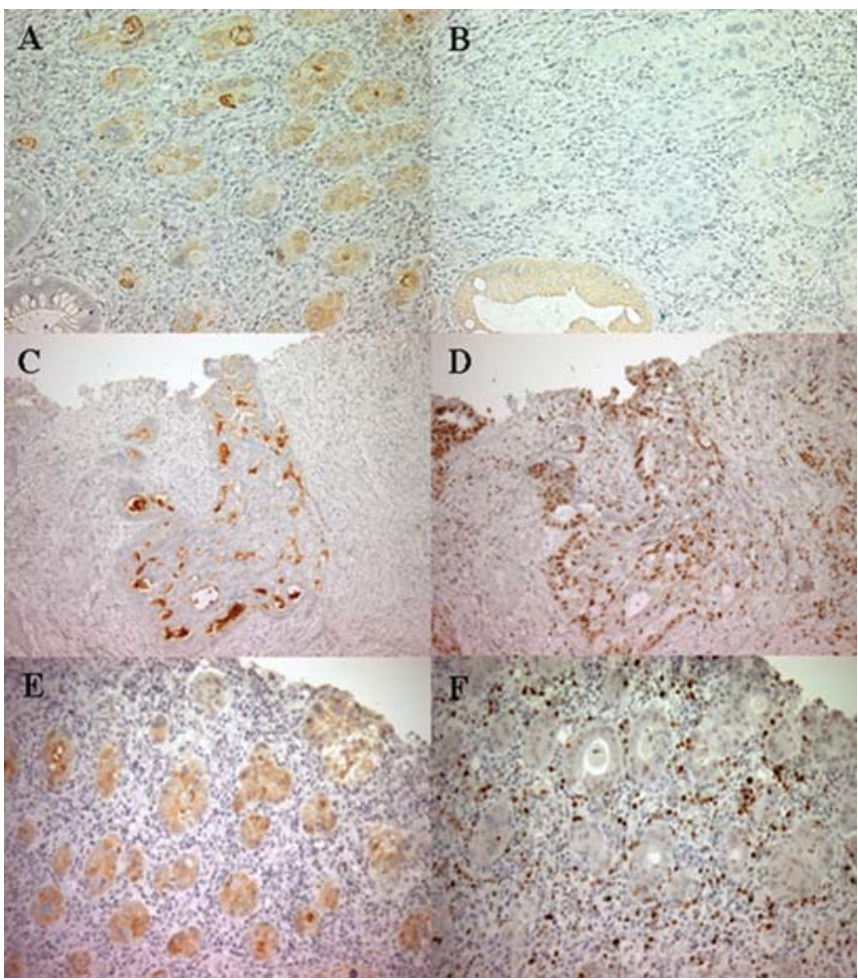

Figure 3. (A) Cytoplasmic CD133-positive cells are scattered around residual cancer after CRT. (B) Cytoplasmic CD133-positive cells lack CK20 expression. (C) Pattern 1 of CD133 staining is shown. (D) Ki-67 is present in residual cancer cells with pattern 1. (E) Cytoplasmic CD133 staining. (F) Ki-67 expression is not detected in cytoplasmic CD133-positive cells. Original magnification, $\mathrm{x} 100(\mathrm{C}$ and $\mathrm{D})$, and $\mathrm{x} 200$ (A, B, E and F).

contents expressing CD133 in small gland-like structures were observed in some specimens (Fig. 2C). CD133 expression in similar deposits was observed in conjunction with intraluminal cells arranged in gland-like structures also expressing CD133. This staining corresponds to pattern 2 (Fig. 2D and E). Fig. 2F-I shows staining patterns 3 and 4. Cytoplasmic staining, including granules, is clearly observed. Residual cancer cells with cytoplasmic CD133 expression in the luminal surface were also observed. CK20 and $\mathrm{Ki}-67$ were present in residual cancer cells with pattern 1 . In contrast, residual cancer cells with cytoplasmic CD133 expression lacked CK20 and Ki-67 staining (Fig. 3).

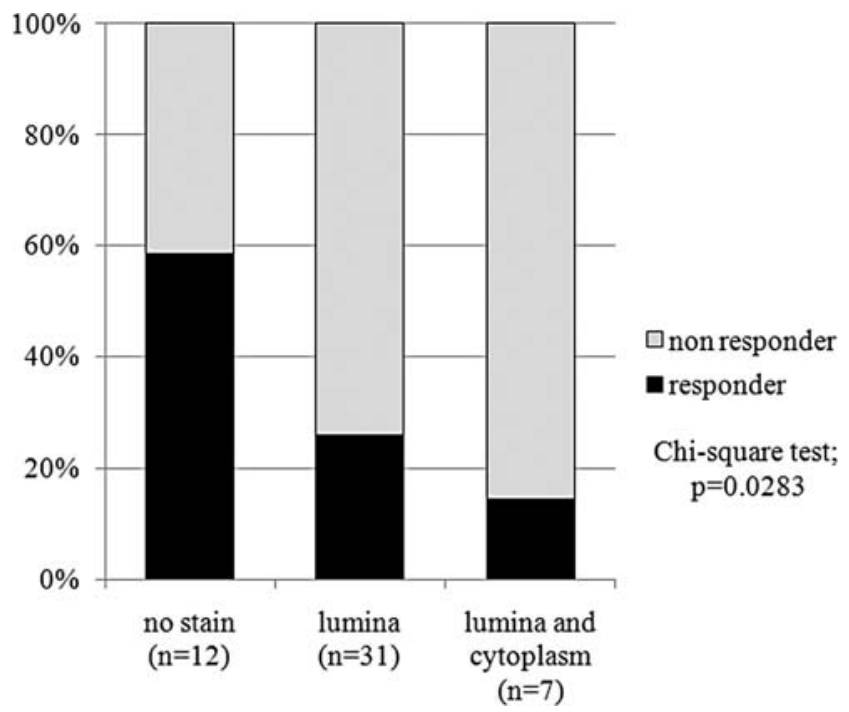

Figure 4. Correlation between the pattern of CD133 expression and histopathological response. Specimens with luminal and cytoplasmic CD133 staining have more non-responders than those without this staining.

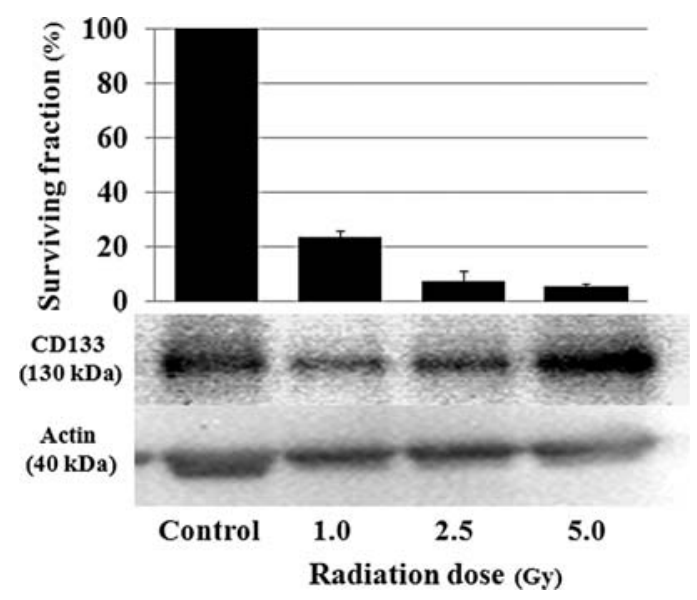

Figure 5. Clonogenic survival assay and expression of CD133 of radiationtreated HT-29 cells. CD133 protein is increased in radiation-dependent manner after irradiation in spite of the decrease of clonogenic survival.

Correlation of CD133 expression patterns with histopathological response. We found histopathologically 16 responders and 34 non-responders in CRT specimens. Fig. 4 shows the correlation between the pattern of CD133 expression and histopathological response after CRT. The ratio of histopathological responder in specimens with CD133 expression in both luminal surface and cytoplasm was significantly lower than that without it $(\mathrm{P}=0.028)$. However, we did not find a correlation between immunohistochemical findings and other clinical variables including distant recurrence and survival (data not shown).

Clonogenic survival and CD133 expression after irradiation in HT-29 cells. As shown in Fig. 5, the reduction of clonogenic survival was observed in radiation-treated HT-29 cells. Survival fraction was reduced in radiation dose-dependent manner. By contrast, single dose of $2.5 \mathrm{~Gy}$ and $5 \mathrm{~Gy}$ irradiation 
increased CD133 protein levels, compared with control. Densitometric analysis showed that CD133 was 1.4 times increased at 5 Gy radiation with respect to control.

\section{Discussion}

Expression of the surface protein CD133 is one criterion that has been used to identify putative CSCs in solid tumors, including colon cancer (5-7). CD133 expression is known to be present in cytoplasm or on the luminal surface, although the function of CD133 remains unclear $(3,6,14)$. In recent studies, the rate of CD133 positive staining was reported to be $15.3-24.5 \%$ in colorectal cancer (17-19). We detected CD133 staining in $27.5 \%$ of non-CRT specimens and $70 \%$ of CRT specimens. Although we understand that CD133 is considered to be a specific marker for colorectal cancer cells because no CD133 staining is found on colonic normal epithelial cells, we think it problematic to confine CD133 to be a specific marker for CSCs in our study. Considering a recent report that $\mathrm{CD} 133$ positive cells exist in $2.0-2.5 \%$ of cancer cells, the frequency of CD133 expression was considerably high (6). This finding is similar to other studies $(8,14,18)$. Especially in CRT specimens, CD133 staining, including luminal surfaces and cytoplasm, was detected in 35 of 50 specimens. We think the reason for such a high frequency of CD133 expression in CRT specimens is that CD133 expression may be up-regulated by CRT because we demonstrated that CD133 protein level was increased in radiation-dependent manner in vitro study. Additionally, in our previous study, CD133 mRNA levels in rectal cancer specimens after CRT were significantly higher than before CRT, and CD133 mRNA levels were also elevated by CRT in an in vitro study $(12,13)$.

To our knowledge, there are few reports of cytoplasmic CD133 staining in colorectal cancer. Immervoll et al reported that cytoplasmic CD133 staining was seen in less than $1 \%$ of the malignant epithelial cells in pancreatic cancer (14), therefore, cancer cells expressing CD133 in cytoplasm are a minor population. In the present study, we detected cytoplasmic CD133 staining clearly in 7\% of CRT specimens, although it was not detected in non-CRT specimens.

We found that residual cancer cells with cytoplasmic CD133 staining had the following features: first, these cells had a regular gland-like structure such as a single duct; second, these cells did not express the differentiated epithelial marker CK20 or the proliferation marker Ki-67. Morphologically, the structures made by cytoplasmic CD133-positive cells seem to be different from those of primary cancer because the structure is in order and isolation, and composed of small numbers of cells. Moreover, we suspect that a structure like the cluster in Fig. 3I is spherical in formation. This structure might be implicated in the process of proliferation or regrowth after CRT, and also the mechanism of protection against CRT.

Several authors also have reported that CD133 positive cells are negative for epithelial markers such as CK19 and CK20 $(6,14,19)$. These findings are suggestive that these cells are an immature or undifferentiated phenotype, and there is a possibility that residual cancer cells with cytoplasmic CD133 staining absent of CK20 are putative CSCs. Considering that
CD133-positive cells have stem cell-like properties such as self-renewal and the ability to differentiate into multiple cell types, CSCs might be immature or undifferentiated.

On the other hand, we found residual cancer cells with cytoplasmic CD133 staining lacking Ki-67 in some CRT specimens. Because the Ki-67 protein is present during all active phases of the cell cycle (G1, S, G2, and mitosis) except for G0, it suggests that these cells are in G0 phase, or cell cycle arrest. Although Kojima et al noted the possibility that CD133 positive cells might be increased only during tumor progression, as one possibility, this might be the mechanism by which CD133 contributes to resistance to radiation (18). Recently, Jaksch et al reported that cell cycle progression is associated with alteration of the CD133 epitope in an in vitro study (20). We think that CD133 positive cells have a reduced sensitivity to radiation and survive radiation damage by inducing cell cycle arrest or cell dormancy in addition to the previous suggestions, such as the activation of DNA damage repair. We found a correlation between CD133 expression and histopathological response to preoperative CRT. This result also suggested that CD133 was associated with resistance to CRT.

In the present study, we did not find an association between CD133 staining patterns and clinicopathological variables, except for histopathological response, because this study included a small number of samples. We plan to investigate using larger numbers of specimens.

In conclusion, we identified various staining patterns of CD133 in rectal cancer after CRT and found that CD133 protein was increased by irradiation. Although the function of CD133 has yet to be elucidated fully, our results suggest that further study of CD133 may provide new findings and will help clarify the role of CD133 in tumor re-growth and resistance to $\mathrm{CRT}$ in colorectal cancer.

\section{References}

1. O'Brien CA, Pollett A, Gallinger S, et al: A human colon cancer cell capable of initiating tumour growth in immunodeficient mice. Nature 445: 106-110, 2007.

2. Polyak K and Hahn WC: Roots and stems: stem cells in cancer. Nat Med 12: 296-300, 2006.

3. Visvader JE and Lindeman GJ: Cancer stem cells in solid tumours: accumulating evidence and unresolved questions. Nat Rev Cancer 8: 755-768, 2008.

4. Vermeulen L, Sprick MR, Kemper K, et al: Cancer stem cells, old concepts, new insights. Cell Death Differ 15: 947-958, 2008.

5. Boman BM and Huang E: Human colon cancer stem cells: a new paradigm in gastrointestinal oncology. J Clin Oncol 26: 2828-2838, 2008.

6. Ricci-Vitiani L, Lombardi DG, Pilozzi E, et al: Identification and expansion of human colon-cancer-initiating cells. Nature 445: 111-115, 2007.

7. Ieta K, Tanaka F, Haraguchi N, et al: Biological and genetic characteristics of tumor-initiating cells in colon cancer. Ann Surg Oncol 15: 638-648, 2008.

8. Shmelkov SV, Butler JM, Hooper AT, et al: CD133 expression is not restricted to stem cells, and both $\mathrm{CD}_{133^{+}}$and $\mathrm{CD} 133^{-}$ metastatic colon cancer cells initiate tumors. J Clin Invest 118 : 2111-2120, 2008.

9. Bao S, Wu Q, McLendon RE, et al: Glioma stem cells promote radioresistance by preferential activation of the DNA damage response. Nature 444: 756-760, 2006.

10. Rich JN: Cancer stem cells in radiation resistance. Cancer Res 67: 8980-8984, 2007.

11. Todaro M, Alea MP, Di Stefano AB, et al: Colon cancer stem cells dictate tumor growth and resist cell death by production of interleukin-4. Cell Stem Cell 1: 389-402, 2007. 
12. Saigusa S, Tanaka K, Toiyama Y, et al: Correlation of CD133, OCT4, and SOX2 in rectal cancer and their association with distant recurrence after chemoradiotherapy. Ann Surg Oncol 16: 3488-3498, 2009.

13. Yasuda H, Tanaka K, Saigusa S, et al: Elevated CD133, but not VEGF or EGFR, as a predictive marker of distant recurrence after preoperative chemoradiotherapy in rectal cancer. Oncol Rep 22: 709-717, 2009.

14. Immervoll H, Hoem D, Sakariassen PO, et al: Expression of the 'stem cell marker' CD133 in pancreas and pancreatic ductal adenocarcinomas. BMC Cancer 8: 48, 2008.

15. Yoshikawa R, Kusunoki M, Yanagi H, et al: Dual antitumor effects of 5-fluorouracil on the cell cycle in colorectal carcinoma cells: a novel target mechanism concept for pharmacokinetic modulating chemotherapy. Cancer Res 61: 1029-1037, 2001.

16. Japanese Society for Cancer of the Colon and Rectum. General Rules for Clinical and Pathological Studies on Cancer of the Colon, Rectum, and Anus. Tokyo, Kanehara \& Co., 2006.
17. Choi D, Lee HW, Hur KY, et al: Cancer stem cell markers CD133 and CD24 correlate with invasiveness and differentiation in colorectal adenocarcinoma. World J Gastroenterol 15: 2258-2264, 2009.

18. Kojima M, Ishii G, Atsumi N, et al: Immunohistochemical detection of CD133 expression in colorectal cancer: a clinicopathological study. Cancer Sci 99: 1578-1583, 2008.

19. Horst D, Kriegl L, Engel J, et al: CD133 expression is an independent prognostic marker for low survival in colorectal cancer. Br J Cancer 99: 1285-1289, 2008.

20. Jaksch M, Munera J, Bajpai R, et al: Cell cycle-dependent variation of a CD133 epitope in human embryonic stem cell, colon cancer, and melanoma cell lines. Cancer Res 68: 7882-7886, 2008. 